\title{
On the Computational Neurobiology of Curve Detection
}

\author{
Steven W. Zucker *Allan Dobbins \& Lee Iverson \\ McGill University Research Center for Intelligent Machines \\ 3480 University St \\ Montreal, Quebec, Canada
}

\begin{abstract}
We deomonstrate the potential for fruitful interaction between those studying artificial vision systems and those studying biological vision systems with the task of inferring global curves with corners and bifurcations from images. The talk outlines one slice through a large project on the computational neurobiology of vision.
\end{abstract}

\section{The Dilemma of Curve Detec- tion}

The initial measurement of orientation information is broadly tuned, which suggests the averaging necessary to counteract retinal (sensor) sampling, quantization, and noise. However, the end result of curve detection is unexpectedly precise: corners can be distinguished from arcs of high curvature, and nearby curves can be distinguished from one another to a hyperaccurate level, even though they might pass through the same receptive field.

An analogous dilemma exists for computer vision systems, even with the spectacular numerical precision of which computers are capable: quantization and noise imply smoothing, but smoothing blurs corners, endpoints, and nearby curves into confusion (Zucker, 1986). At the foundation is a chicken-and-egg problem: if the points through which the curve passed, together with the locations of discontinuities, were known, then the actual properties of the curve could be inferred. But initially they are not known, so any smoothing inherent in the inference process is potentially dangerous.

\section{Two Stages of Curve Detection}

We have discovered a computational solution to this dilemma, which involves decomposing the full problem into two stages, each of which has a rather different character (Fig. 1). Our approach differs significantly

\footnotetext{
*and Canadian Institute for Advanced Research
}

from previous ones in that no attempt is made to match curves directly against the image, or even against measurements (i.e., "curve operators") evaluated over the image. Instead we stress the organizational point that a stable, reliable, but coarse description should be computed as an intermediate between the image (measurements) and the global curves. A particular intermediate representation - the discrete tangent field-is proposed.

The second stage of the algorithm synthesizes the global curves through the tangent field. The novel idea behind our approach is to recover the global curve by computing a covering of it; i.e., a set of objects whose union is equivalent to the original curve, rather than attempting to compute the global curve directly. The elements of the covering are unit-length dynamic splines, and global curves are recovered to sub-pixel accuracy.

\section{The Model of Curve Detection}

We define orientation selection to be the inference of a local description of the curve everywhere along it, and postulate orientation selection as the goal of our first stage. In the second stage, global curves are inferred through this local description. The various stages of our process are shown in Fig. 1, and expanded below.

\subsection{Stage 1: Inferring the tangent field}

Formally orientation selection amounts to inferring the trace of the curve, or the set of points (in the image) through which the curve passes, its (approximate) tangent and curvature at those points, and their discontinuities [Zucker, 1986]. We refer to such information as the tangent field, and note that, since the initial measurements are discrete, this will impose constraints on the (inferred) tangents, curvatures, and discontinuities [Parent and Zucker, 1988].

This first stage of orientation selection is in turn modeled as a two step process:

- Step 1.1. Initial Measurement of the local fit at each point to estimate orientation and curvature. These 


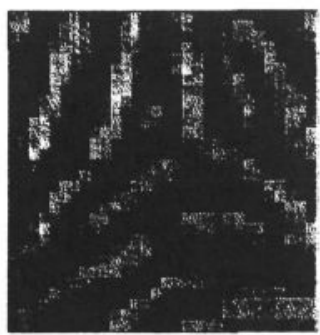

(a)

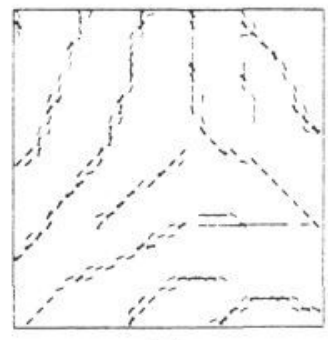

(c)

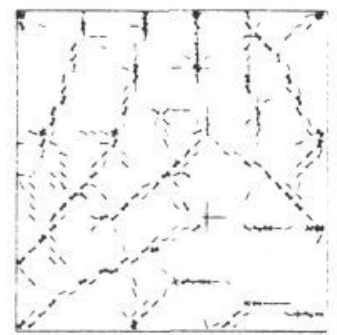

(b)

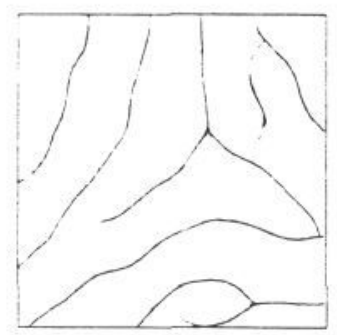

(d)

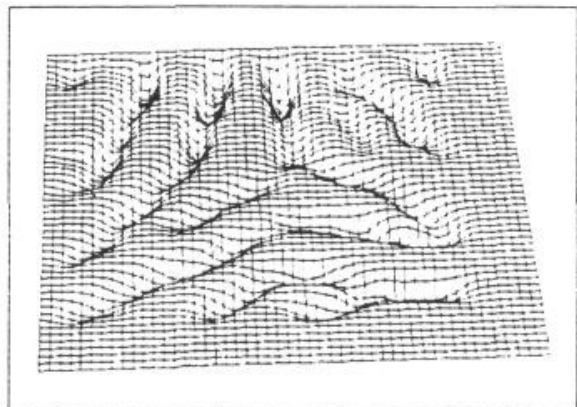

(e)

Figure 1: An illustration of the different stages of curve detection. In the first stage, a reliable, but coarse, description (tangent field) of the local structure of curves is computed, while global structure is computed in the second. This is illustrated for the small fingerprint image (a); note the smooth curves and discontinuities around the " $Y$ " in the center. The first stage is broken into two steps. In the first step, initial (non-linear) measurements are performed to estimate what the local curvature and orientation might be (b). In the second step these initial measurements are refined by a relaxation labeling process and (c) shows the final tangent field after 2 iterations; note that most of the spurious initial responses have been eliminated. There are also two steps in the second stage, first the construction of a potential distribution from the entries in the tangent field (e), and second, the covering of the global curve by a family of short curves, or snakes (d). 
estimates derive from a model of simple cell receptive fields instantiated at multiple scales and orientations at each image position. The model is nonlinear, and derives from a sub-field partition and then re-combination provided certain (Boolean) logical preconditions are satisfied [Iverson and Zucker, 1990]. The result eliminates many of the false positive responses that plague other operators. Curvature estimates are then derived from non-linear differences between orientation measurements at different scales, and is related to the physiological property of endstopping [Dobbins, Zucker, and Cynader, $1987 ; 1989]$. We thus propose that endstopped neurons in the visual cortex represent joint hypotheses about orientation and curvature, and that their firing rate along an (endstopped) orientation hypercolumn represents how well these hypotheses match the local image structure. However, such local measurements are inherently inaccurate (e.g., broadly tuned), so we require

- Step 1.2. Interpretation into an explicit distributed representation of tangent and curvature by establishing consistency between the local measurements. Consider an arc of a curve, and observe that tangents to this arc must conform to certain position and orientation constraints for a given amount of curvature; we refer to such constraints geometrically as co-circularity (Fig. 2a). Discretizing all continuous curves in the world that project into the columnar space of coarse (orientation, curvature) hypotheses partitions these curves into equivalence classes, (Parent and Zucker, 1989; Zucker and Iverson, 1988). Interpreting the (orientation, curvature) hypotheses as endstopped neurons, such cocircularly-consistent relationships are expected between endstopped neurons in nearby orientation hypercolumns given such a curve as stimulus.

Such inter-columnar interactions can be viewed physiologically as excitatory and inhibitory projections between endstopped cells at nearby positions (adjacent hypercolumns), and can be used as follows. Since curvature is a relationship between tangents at nearby positions, two tangents should support one another if and only if they agree under a curvature hypothesis, and cocircularity provides the measure of such support. In addition, two tangents that disagree with the curvature estimate should detract support from one another. Relaxation labeling provides a formal mechanism for defining such support, and for specifying how to use it [Hummel and Zucker, 1983]. Mathematically it amounts to gradient descent; physiologically it can be viewed as the computation implemented by pyramidal neurons as they combine information from adjacent (endstopped) orientation hypercolumns. Since only $2-3$ iterations are required for convergence (empirically), it is natural to propose that these are accomplished by the forward- and back-projecting pyramidal neurons connecting areas V1 and V2 [Zucker et al., 1989].

One specific model of the physiology could be as follows: initial orientation estimates are obtained by the small (layer 4) simple cells in V1; the difference required for curvature estimation can be built up from a layer 6 to layer 4 back projection, and the first iteration of the relaxation process can be implemented by a pyramidal neuron with dendritic inputs from nearby (endstopped) orientation hypercolumns. Such neurons project to V2, where a similar computation takes place; the backprojection to V1 then provides a final iteration. The tangent field is represented by those neurons whose firing rate is maintained, with tangent corresponding to preferred orientation, and curvature to percentage endstopping.

Similar ideas can be applied to texture, suggesting a difference between texture flows, or those texture patterns with an orientation structure, and texture fields, e.g. "salt-and-pepper" patterns [Hel Or and Zucker, 1989]. The extension into three dimensions requires more sophisticated differential geometry; see Sander and Zucker [1990]. Also, application to laser rangefinder imagary is very successful [Ferrie et al., 1990].

\subsection{Stage 2: Inferring a Covering of the Curve}

Since the tangent is the first derivative of a curve (with respect to arc length), the global curve can be recovered as an integral through the tangent field. Such a view typically leads to sequential recovery algorithms (e.g., Kass and Witkin [1987]). But these algorithms require global parameters (e.g., total length), starting points, and some amount of topological structure (i.e., which tangent point follows which); in short, they are biologically implausible. In contrast, we propose a novel approach in which a collection of short, dynamically modifiable curves ("snakes" in computer vision; see Kass, Witkin and Terzopoulos [1988]) move in parallel.

Recovering the global curve by computing a covering of it; i.e., a set of objects whose union is equivalent to the original curve, avoids the prerequisite global problems. Let the elements of the covering be unit-length dynamic splines, initially equivalent to the elements of the tangent field, but which then evolve according to a potential distribution constructed from the tangent field. The evolution takes two forms: (i) a migration in position to achieve smooth coverings; and (ii) a "growth" to triple their initial length.

Again, there are two conceptually distinct steps to Stage 2 of the algorithm: 

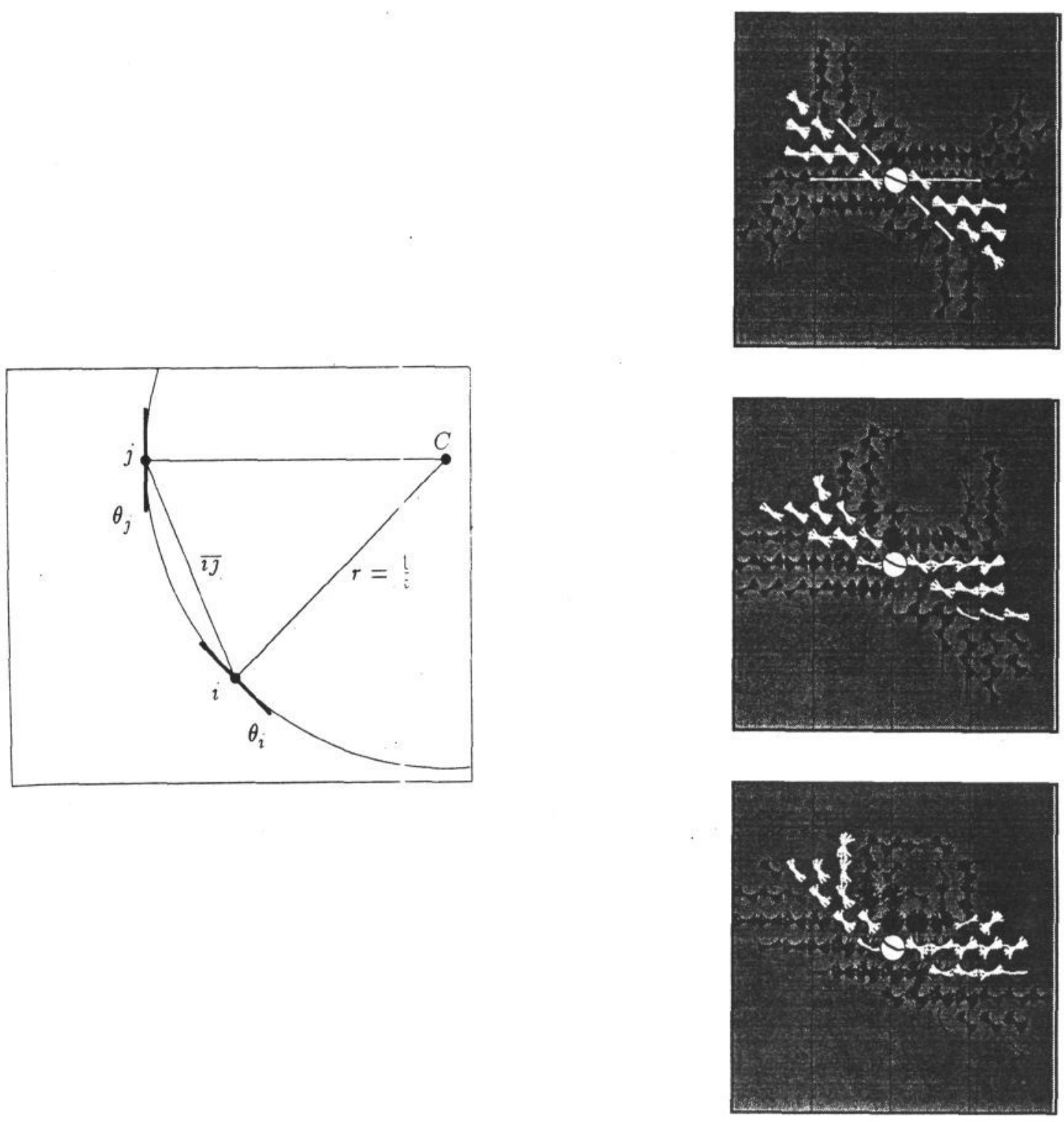

Figure 2: (a)The geometric relationships necessary for defining the compatibilities between two label pairs at nearby points $i=\left(x_{i}, y_{i}\right)$ and $j=\left(x_{j}, y_{j}\right)$. (b) Compatibilities between coarse (orientation, curvature) hypotheses at nearby positions. 8 distinct orientations and 7 curvatures are represented, and 3 examples are shown. (top) The labels which give positive (white) and negative (black) support for a diagonal orientation with no curvature; (middle) positive and negative support for a small curvature class; (bottom) positive and negative support for the maximum curvature class. The magnitude of the interactions varies as well, roughly as a Gaussian superimposed on these diagrams. The values were obtained by numerically solving a 6-dimensional closest point problem (Zucker et al., 1988). Physiologically these projective fields could represent inter-columnar interactions implemented by pyramidal neurons. 
- Step 2.1. Constructing the Potential Distribution from the discrete tangent field. Each entry in the tangent field actually represents a discretization of the many possible curves in the world that could project onto that particular (tangent, curvature) hypothesis. Now these pieces must be put together. Assuming the curves are continuous but not necessarily differentiable everywhere, each contribution to the potential can be modeled as a Gaussian (the Wiener measure) oriented in the direction of the tangent field entry. The full potential distribution is their pointwise sum; see Fig. 3.

- Step 2.2. Spline Dynamics The discrete entities in the tangent field are converted into unit splines initialized in the valleys of the potential distribution. They evolve according to a variational scheme that depends on spline properties (tension and rigidity) as well as the global potential (Fig. 3).

The potential distribution is created by adding together contributions from each element in the tangent field; see Fig. 3. Changing the representation from the tangent field to the potential distribution changes what is explicit and what is implicit in the representation, and local information is combined into global information. In Stage 1 there were discrete coarse entities; now there are smooth valleys that surround each of the global curves, with a separation between them. The "jaggies" imposed by the initial image sampling have been eliminated, and interpolation to sub-pixel resolution is viable.

To recover the curves through the valleys, imagine creating, at each tangent field entry, a small spline of unit length oriented according to the tangent and curvature estimates (Fig. 4). Since each spline is born in a valley of the tangent field potential distribution, they are then permitted to migrate to both smooth out the curve and to find the true local minima. The union of these local splines is the global cover. But the the splines must overlap, so that each point on every curve is covered by at least one spline. We therefore let the splines extend in length while they migrate in position, until they each reach a prescribed length. The covering is thus composed of these extensible splines which have grown in the valleys of the tangent field potential. Their specific dynamics and properties are described more fully in Zucker et al. (1988); David and Zucker (1988).

It is difficult to interpret these ideas physiologically within the classical view of neurons, in which inputs are summed and transformed into an output train of action potentials. Dendrites simply support passive diffusion of depolarization. Recently, however, a richer view of neuronal processing has emerged, with a variety of evidence pointing to active dendritic computation and dendrodendritic interaction [Schmitt and Worden, 1979]. Active conductances in dendrites functionally modify the geometry, and dendro-dendritic interactions suggest that the output transformation is not uniquely mediated by the axon. Taken together, these facts imply that patterns of activity can be sustained in the dendritic arbor, and that this membrane could be the substrate of the above potential distribution computations.

The large constructed potential distributions may bear some resemblance to the large receptive fields observed in areas V4 and IT [Maunsell and Newsome, 1987]. While any such relationship is clearly speculative at this time, it should be noted that they have two key similarities: (i) extremely large receptive fields (potential distributions) have been created, but they maintain about the same orientation selectivity as in V1; (ii) their structure can change. We have stressed how structure is controlled by upward flowing information, but it should also be modifiable by "top-down" attentional influences as well [Maunsell and Newsome, 1987; Moran and Desimone, 1985]. Attention could easily "gate" the tangent field entries at the creation of the potential.

\section{References}

David, C., and Zucker, S.W., Potentials, valleys, and dynamic global coverings, Tech. Rep. TR-CIM-891, McGill Research Center for Intelligent Machines, Montreal, 1989; Int. J. Computer Vision, in press.

Desimone, R., Schein, S., Moran, J., and Ungerleider, L., Contour, color, and shape analysis beyond the striate cortex, Vision Research, 1985, 25, 441 - 452.

Dobbins, A., Zucker, S.W., \& Cynader, M.S. (1987) Endstopping in the visual cortex as a substrate for calculating curvature. Nature, 329, 438-441, 1987.

Dobbins, A., Zucker, S.IV., and Cynader, M., Endstopping and curvature, Vision Research, 1989, 29, $1371-1387$.

Ferrie, F.P., and Lagarde, J., Whaite, P., Darboux Frames, Snakes, and Super-Quadrics: Geometry From the Bottom-Up, IEEE Workshop on Interpretation of $3 D$ Scenes, Austin, Texas, November 27-29, 1989, pp. 170-176.

Ferrie, F.P., and Lagarde, J., Whaite, P., Recovery of Volumetric Object Descriptions From Laser Rangefinder Images, First European Conference on Computer Vision, Antibes, France, April 1990.

Nelson, J.J. \& Frost, B.J. (1985) Intracortical facilitation 

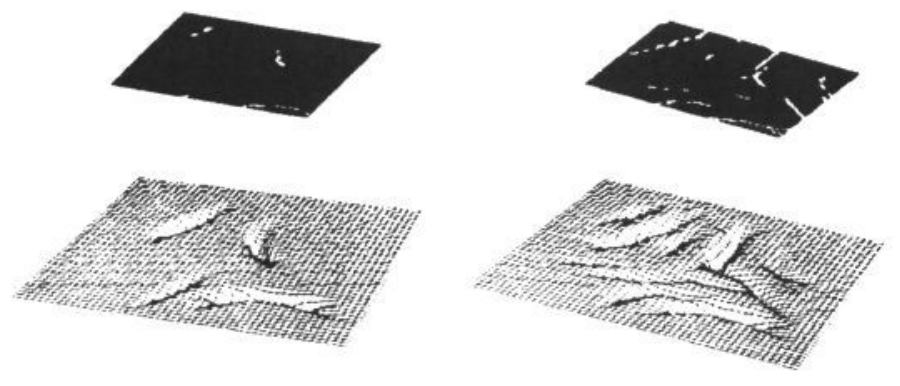

Figure 3: Illustration of how a potential distribution is constructed from tangent field entries. (a) A small number of tangents, showing the individual contributions from each one. (b) As more tangents are included, long "valleys" begin to form when the individual entries are added together. The complete tangent field and potential distribution are shown in Fig. 1. Physiologically one might think of such potentials as being mapped onto local clusters of neuronal membranes, and supported by localized dendric or dendro-dendritic interactions.

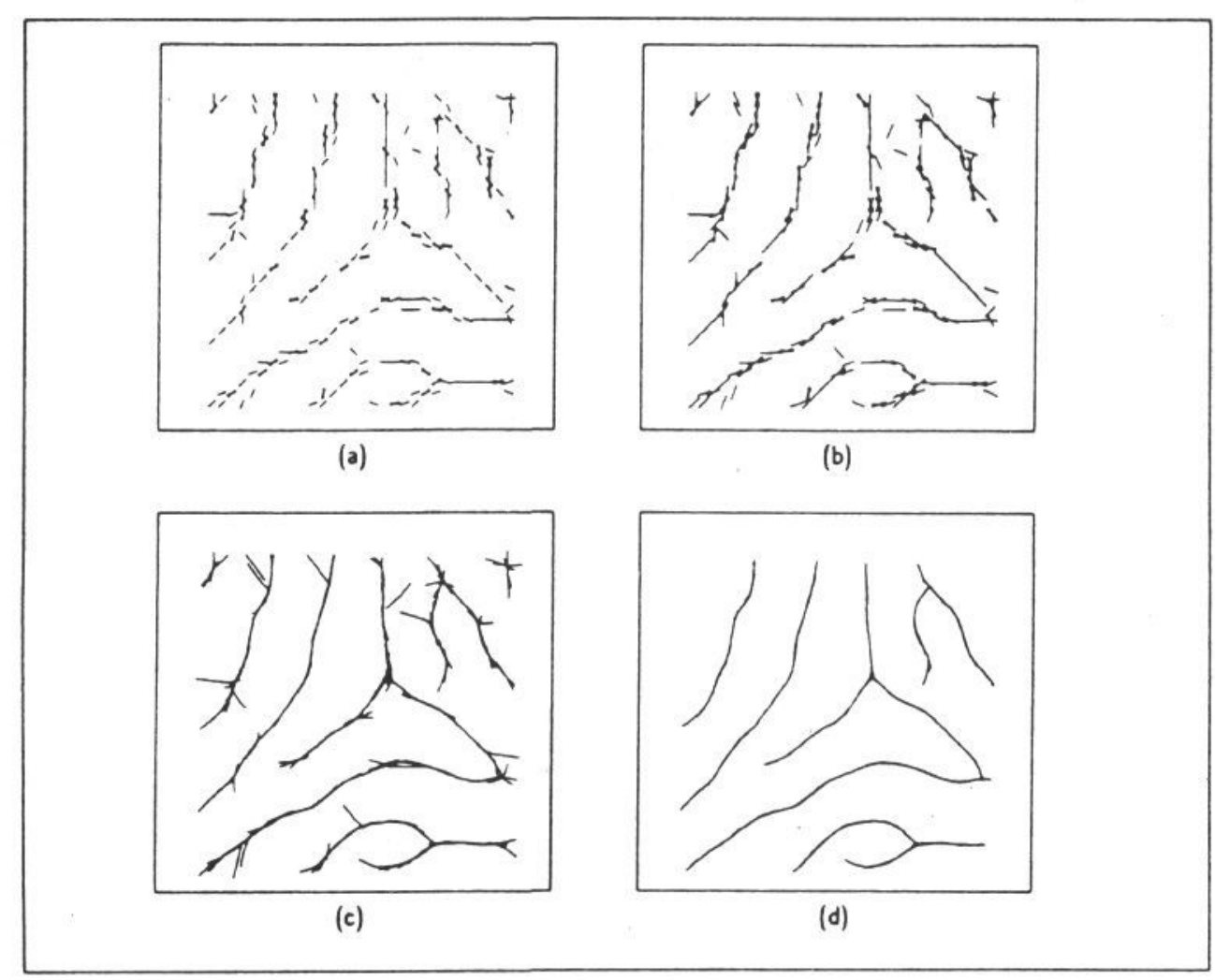

Figure 4: Illustration of the splines in motion. Initially, each spline is born at a tangent field location, with unit length. Then, according to the potential distribution shown in Fig. 1e, the splines migrate in position (to find minima in the distribution) and in length, so that they overlap and fill in short gaps. At convergence, the length of each spline has tripled. 
among co-oriented, co-axially aligned simple cells in cat striate cortex. Exp. Br. Res., 61: 54-61.

Hel Or, Y., and Zucker, S.W., Texture fields and texture flows: Sensitivity to differences, Spatial Vision, 1989, 4, 131 - 139.

Hummel, R., and Zucker, S.W., On the foundations of relaxation labelling processes, IEEE Trans. PAMI 5, 1983, pp. 267-287.

Iverson, Lee, and Zucker, S.W., Logical/Linear operators for measuring orientation and curvature, Technical Report 90-6, Research Center for Intelligent Machines, McGill University, 1990.

Kass, M., Witkin, A., and Terzopoulos, D., SNAKES: active contour models, Int. J. Computer Vision, 1988, 1, 321 - 332 .

Maunsell, J., and Newsome, W., Visual processing in monkey extrastriate cortex, Ann. Rev. Neuroscience, 1987, 10, 363 - 401 .

Moran, J., and Desimone, R., Selective attention gates visual processing in the extriastriate cortex, Science, 1985, 229, 782 - 784.

Parent, P. and Zucker, S.W., Trace Inference, Curvature Consistency, and Curve Detection, IEEE Trans. Pattern Analysis and Machine Intelligence, 1989, 11, $823-839$.

Sander, P., and Zucker, S.W., Inferring differential structure from 3-D images: Smooth cross sections of fibre bundles, IEEE Trans. Pattern Analysis and Machine Intelligence, in press.

Sander, P., and Zucker, S.W., Singularities of principal direction fields from 3-D images, IEEE Trans. Pattern Analysis and Machine Intelligence, in press.

Schmitt, F., and Worden, F. (eds.), The Neurosciences: Fourth Study.Program, MIT Press, Cambridge, 1979 .

Zucker, S.W., David, C., Dobbins, A., and Iverson, L., The organization of curve detection: Coarse tangent fields and fine spline coverings, Proc. $2^{\text {nd }}$ Int. Conf. on Computer Vision, Tarpon Springs, Fla, 1988.

Zucker, S.W., and Iverson, L., Computational networks in early vision: From orientation selection to optical flow, in Eckmiller, R., and v.d. Malsberg, C. (eds.), Neural Computers, Springer, New York, 1988 .

Zucker,S.W., Dobbins, A., and Iverson, L., Two stages of curve detection suggest two styles of visual computation, Neural Computation, 1989, 1, 68-81. 
